\title{
Some models of observer behavior in two-channel auditory signal detection*
}

\author{
R. D. SORKIN and L. D. POHLMANN \\ Purdue Linilersitv: Lafavette. Indiana 47907
}

\begin{abstract}
Five models of $O$ behavior in a two-channel exclusive-or (XOR) detection task were evaluated. The models included two types of single-channel and three types of two-channel $O$. Only the most efficient two-channel model adequately described human performance in a set of monaural and dichotic XOR conditions. Detectability measures derived from the XOR task matched those obtained from separate single-channel control conditions. We concluded that, in this two-channel task, the $O$ 's performance was not limited by any inability to monitor signals arriving simultaneously in two different earphone or two different frequency channels. The implications of this result for two-channel information processing and for multicomponent and sequential signal detection are discussed.
\end{abstract}

Many scientists, including Moray (1970a, b), have argued that performance decrements in two-channel listening tasks reflect an O's inability to monitor two auditory channels at the same time. Although our previous experiments have demonstrated performance decrements in two-channel detection tasks (Sorkin, Pohlmann, \& Gilliom, 1973), we suggested that the decrement was caused by interference between the channels that was generated when an occasional intense input occurred in one channel. In the present paper, we evaluate five models of two-channel signal detection in a type of task in which cross-channel interference is expected to have a minimal effect.

Our previous experiments employed different classes of signals but the same basic two-channel task. On each trial of the experiment, the $\mathrm{O}$ had to report whether a signal was present in Channel A, Channel B. both channels. or neither of two defined signal channels. The signals were presented independently in each channel: "channel" was defined as the earphone channel or the frequency band that might contain the signal. The signals always occurred with probability equal to $1 / 2$ within each defined channel. The types of signals employed included pure tone bursts in each earphone (Pastore \& Sorkin, 1972; Sorkin \& Pastore, 1971). narrow-band noise bursts of differing interaural correlation in each earphone (Sorkin, Pastore, \& Pohlmann, 1972), and pure tone bursts of differing frequency in the same and in different earphone channels (Sorkin, Pohimann, \& Gilliom, 1973). Because signal occurrence in each channel was independent of the other channel, it was possible to compute separately the detectability of the signal in each channel and to compare that value with detectability in an appropriate single-channel control condition. In addition, it was possible to compute the detectability of any channel conditional upon specific signal and response events in

\footnotetext{
*This research was partially supported by a grant from the National Science Foundation. The authors thank M. Treisman and R. Pastore for many useful comments on an earlier draft of this paper.
}

the other channel, such as signal vs no signal and correct vs incorrect. The results of these experiments can be simply summarized. In addition to known monaural and binaural detection phenomena, we observed a cross-channel interference effect: performance in one channel was degraded on trials when a signal had occurred in the other channel. This effect was large enough to account for all of the differences between the two-channel and single-channel performance levels. Apparently, large observations made in one channel can lead to an interruption of "normal" processing in the other channel.

In a report of related work in our laboratory, Gilliom (1972) suggested an analogy between the simultaneous monitoring of several channels and a computer in an interrupt model of operation. The $O$ is assumed to simultaneously monitor the two channels and to continuously integrate the energy output of each auditory channel. When the output of any of the channels reaches some critical value, an interrupt is generated. At this point, integration is terminated and the measures made on each channel are processed sequentially, with some finite time required for processing the information in each channel. Since the channel that generated the interrupt is processed first. there is some degrading of the information obtained in the other channel. This view is similar to the hypothesis that inputs occurring in one channel may cause an attenuation or gating of ongoing processing in the other channel (Broadbent, 1971; Moray, 1969; A. Treisman, 1969).

Many investigators, including Sorkin (1965), Taylor, Clarke, and Smith (1971), M. Treisman (1963). Yost, Penner, and Feth (1972), and Zwislocki. Damianopoulos, Buining, and Glantz (1967), have noted cross-channel masking effects that are dependent on the intensity and frequency of the signal in the contralateral earphone channel. However, the cross-channel interference we observed in our most recent study (Sorkin, Pohlmann, \& Gilliom, 1973) did not match the data on contralateral masking. In that study. there was (1) interference between widely separated frequency 
bands. and (2) no decrease in this interference when the frequency bands were presented to the separate earphone channels rather than monaurally. We concluded that this interference was not consistent with a contralateral masking interpretation.

The goal of the present experiment was to evaluate behavior in a type of two-channel detection task in which this cross-channel effect should be minimized or eliminated. Such a task has been employed by Moray $(1970 \mathrm{a}, \mathrm{b})$ in a related set of two-channel auditory detection experiments. In Moray's exclusive-or (XOR) task, the signal could occur in either or neither but not both channels. We will argue that our hypothesized $O$ is not vulnerable to an interrupt-interference effect in the XOR task.

In general, if the observations on both channels exceed the criteria for responding "signal," the O should make the "signal" response associated with the channel having the larger observation (assuming that the detectabilities and signal presentation probabilities in each channel are equal). Let us assume that the observation made on each channel is based on some sort of integration or counting process computed during the observation interval. When the integration process in Channel $A$ reaches some critical value, there is an interruption of computation in Channel $B$ and Channel A. Provided that the critical value for terminating the integration process is relatively large, a vote between the channel observations based on a partial integration will usually agree with a vote based on a full integration. Specifically, if the critical value were large relative to the mean of the distribution of signal observations in that channel, the likelihood of an upset in an interrupted computation would be very small. ${ }^{1}$ Thus, it would be safe to terminate processing as soon as the observation in either channel reached a critical value, and the interrupt-prone $O$ would suffer no decrement in the XOR task. In that case, performance in the XOR task would be predictable from performance in the appropriate single-channel tasks. This result is in contrast to the more general two-channel task where either, neither, or both signals can occur (the inclusive-or task), in which the $\mathrm{O}$ must process both channels sufficiently to make separate yes/no decisions about each channel. The requirement for complete simultaneous processing makes the inclusive-or (IOR) task highly vulnerable to the interruptions caused by large observations in the other channel.

Moray's experiments have included both exclusive-or and inclusive-or conditions and employed increments to sinusoid tone bursts as signals presented in each earphone channel. Moray's tasks differ from our experiments in at least two important ways. First, in his tasks, the $\mathrm{O}$ had to respond to an ongoing stream of tone bursts which were paced at the rate of $2 / \mathrm{sec}$. Thus, his task probably involves factors of attention and memory which are more complex, and perhaps more real-life, than those involved in the standard type of psychophysical situation. Second, in some conditions, the probability of signal occurrence in each channel was dependent on signal occurrence in the other channel. This presentation contingency complicates analysis of the channel detectabilities. Garner and Morton (1969) discussed the measurement problems involved in these and related two-channel paradigms. In a discussion of Moray's study, Treisman (1972) suggested that a rational O might employ his knowledge of the signal presentation contingency to improve his performance. Treisman suggested that the $\mathrm{O}$ might employ two different criteria in each channel, depending on the value of the observation made on the other channel. For such an $\mathrm{O}$, the criterion measured in one channel would be dependent on signal occurrence in the other channel, but there would not necessarily be any effect on the detectability of a channel due to signal occurrence in the remote channel.

In a previous report (Sorkin, Pohlmann, \& Gilliom, 1973), we suggested that an $O$ in an IOR task might employ a range of criteria that were dependent on the magnitude of the observation in the remote channel. The variability of the O's criteria would depend on the variability of the remote channel observation. Increases in criterion variability would be evidenced by decreases in the d' of the channel. In general, we would not expect that this variability would increase as a simple function of the absolute magnitude of the observations in the remote channel; variability would probably be highest for a range of values around some critical observation magnitude in the remote channel. If this critical value was relatively distant from the mean of the noise-alone distribution (in each channel), then decrements due to criterion variability would occur only very rarely in XOR tasks. It may be noted that the use of a criterion in IOR tasks that is dependent on the remote channel observation is a rational strategy only in tasks where there is a contingency between the occurrence of a signal in each channel. In our previous experiments, the Os were informed of the fact that there was no such contingency.

In the XOR task, the data are in the form of a 9-entry confusion matrix (3 stimuli by 3 responses); computation of the individual channel detectabilities from this matrix requires detailed specification of the $O$ 's listening strategy. We propose five simplified models of possible $\mathrm{O}$ behavior in the XOR task. These include two types of single-channel $O$ and three types of two-channel $O$. All of the models include the typical theory of signal detectability (TSD) assumption that the O can compute a continuous measure on the observation made in at least one signal channel (cf. Tanner, 1956; Eijkman \& Vendrik, 1965). For each model, we also assume that observations made on each channel are independent. The mean of the observation distributions, given noise alone, is zero; the mean of the signal plus noise distribution in a channel is assumed to be the $\mathrm{d}^{\prime}$ for that channel. 


\section{Model 1 Single-Channel Observer (SCO)}

This $O$ can monitor only one channel during the observation interval in the XOR task; he monitors his more sensitive channel. If the observation in that channel exceeds a criterion, $\beta_{1}$, he responds " $S_{1}$ "; if the observation does not exceed $\beta_{1}$, he responds " $S_{2}$ " with probability $\alpha$, and " $\bar{S}_{1} \cdot \overline{\mathrm{S}}_{2}$ " with probability $1-\alpha$.

\section{Model 2 Same-Different Observer (SDO)}

This 0 simultaneously monitors both channels, computes a measure on each, $x$ and $y$, and subtracts one measure from the other, $x-y=z$. The value of the difference measure, $z$, is compared to criteria $\beta_{1}$ and $\beta_{2}$. For $z<\beta_{1}$, he responds " $S_{1}$ "; for $z>\beta_{2}$, he responds " $\mathrm{S}_{2}$ "; and for $\beta_{1}<z<\beta_{2}$, he responds " $\overline{\mathrm{S}}_{1} \cdot \overline{\mathrm{S}}_{2}$." This is the model described by Sorkin (1962) for a task requiring same-different judgments on auditory signals. Zinnes and Kurtz (1968) discussed a variety of related models applicable to such tasks. Combining the measures $\mathrm{x}$ and $\mathrm{y}$ in this way is an inappropriate procedure in the XOR task, since the resulting statistic has double the variance of $x$ or $y$ alone; presumably, if the $O$ could compute $z$, he could also employ a more optimal strategy. The simplicity of the resulting one-dimensional normal decision axis is one aspect in favor of the model.

\section{Model 3 \\ Two-Channel Unweighted Observer (TCU)}

This $\mathrm{O}$ can simultaneously monitor both channels, obtaining independent measures, $x$ and $y$, on the respective channels. If $x<\beta_{1}$ and $y<\beta_{2}$, he responds " $\bar{S}_{1} \cdot \bar{S}_{2}$." If either $x$ or $y$, but not both, exceeds its respective criterion, $\beta_{\mathrm{i}}$, he responds with the appropriate channel " $S_{i}$." If both $x$ and $y$ exceed their respective criteria, the $O$ compares $\left(x-\beta_{1}\right)$ with $\left(y-\beta_{2}\right)$ and responds with the signal associated with the higher channel; e.g., if $\left(x-\beta_{1}\right)>\left(y-\beta_{2}\right)$, he responds " $S_{1}$," and if $\left(x-\beta_{1}\right)<\left(y-\beta_{2}\right)$, he responds " $S_{2}$." This $O$ could improve his performance by weighting $x$ and $y$ by their respective single-channel detectabilities, prior to making the $\mathrm{x}, \mathrm{y}$ comparison. For simplicity, we have assumed that they are weighted equally. The decision space for TCU can be visualized as a two-dimensional space that includes the three bivariate normal distributions (for $x, y$ given noise alone, $S_{1}$, and $S_{2}$ ) and three criterion regions. These regions are bounded by two criterion lines at $\beta_{1}$ and $\beta_{2}$ oriented normal to the axes (the channels) and by one criterion line which originates at the intersection of the other two lines and is at a 45-deg orientation to the axes [beginning at $\left(\beta_{1}, \beta_{2}\right)$ with $\left.y=x+\beta_{2}-\beta_{1}\right]$. This model is similar to the "double-detection" strategy model described by
Treisman and Leshowitz (1969) in a report of forced-choice visual discrimination.

\section{Model 4 \\ Two-Channel Flip Observer (TCF)}

This $O$ can also simultaneously monitor both channels and obtain independent measures, $x$ and $y$, on each. If $x<\beta_{1}$ and $y<\beta_{2}$, he responds " $\bar{S}_{1} \cdot \bar{S}_{2}$." If either $x$ or $\mathrm{y}$, but not both, is greater than its respective criterion, the response is the appropriate channel, " $\mathrm{S}_{\mathrm{i}}$." The problem for this $\mathrm{O}$ occurs when both $\mathrm{x}$ and $\mathrm{y}$ exceed their respective criteria. In that case, this $O$ flips a fair coin and responds " $S_{1}$ " with a probability of $1 / 2$ and " $\mathrm{S}_{2}$ " with a probability of $1 / 2$. The performance of this $O$ is dependent on the relative conservatism of the criteria $\beta_{1}, \beta_{2}$. If these criteria are relatively strict, his behavior will approximate that of TCU. As the criteria shift toward the noise distribution, the number of coin flips required will increase, and performance will suffer.

\section{Model 5 \\ Single-Channel Multiplexer (SCM)}

This $O$ is identical to TCU except that he can monitor only one channel at a time. We assume that he can sample both channels in such a way that he has half the duration of each channel on which to compute a measure. His performance, then, will simply be a less efficient version of TCU, with computed single-channel $d^{\prime}$ 's that are $\sqrt{2}$ larger than those of TCU. That is, in order for SCM to produce the same performance (confusion matrix) as TCU. SCM's single-channel $d$ 's would have to be $\sqrt{2}$ larger. This assumes that the effect of halving signal duration follows the normal effect on detectability for the actual signal levels and durations employed.

The appendix includes a derivation of the equations required for computation of the hit and confusion probabilities for the SCO, SDO, TCU, and TCF Os.

\section{METHOD}

The two channels in which the signals might occur were defined as two very narrow frequency regions, one centered at $630 \mathrm{~Hz}$ and the other centered at $1,400 \mathrm{~Hz}$. The basic task throughout the experiment was to respond whether (a) no signal had occurred. (b) the $630-\mathrm{Hz}$ signal had occurred, or (c) the $1,400-\mathrm{Hz}$ signal had occurred. Two variations of this basic task were run which included (a) a monaural presentation condition in which the $630-\mathrm{Hz}$. the $1.400-\mathrm{Hz}$, or neither signal might be present in the $O$ 's left earphone, and (b) a dichotic condition in which the $630-\mathrm{Hz}$ signal could occur only in the left earphone and the $1.400-\mathrm{Hz}$ signal could occur only in the right earphone. In all conditions of the experiment, uncorrelated noise was present in both earphones. In addition to the two-frequency conditions. single-channel control conditions were run separately on the $630-\mathrm{Hz}$ signal in the left ear and on the $1.400-\mathrm{Hz}$ signal in the left and right ears. Table 1 summarizes the XOR and single-channel control conditions. 
Table 1

Summary of Single-Frequency and XOR Conditions

\begin{tabular}{|c|c|c|c|c|}
\hline \multirow[b]{2}{*}{ Condition } & & \multicolumn{2}{|c|}{ Signal Set } & \multirow{2}{*}{$\begin{array}{c}\text { Response } \\
\text { Set }\end{array}$} \\
\hline & & Left & Right & \\
\hline \multirow{3}{*}{ Single-Frequency } & 1 & $\begin{array}{l}\mathrm{N} \\
\mathrm{S}_{1}\end{array}+\mathrm{N}$ & $\begin{array}{l}\mathbf{N} \\
\mathbf{N}\end{array}$ & $\begin{array}{ll}\text { No } & \left(S_{1}\right) \\
\text { Yes } & \left(S_{1}\right)\end{array}$ \\
\hline & 2 & $\begin{array}{l}\mathrm{N} \\
\mathrm{S}_{2}+\mathrm{N}\end{array}$ & $\begin{array}{l}\mathbf{N} \\
\mathbf{N}\end{array}$ & $\begin{array}{ll}\text { No } & \left(S_{2}\right) \\
\text { Yes } & \left(S_{2}\right)\end{array}$ \\
\hline & 3 & $\begin{array}{l}\mathrm{N} \\
\mathrm{N}\end{array}$ & $\stackrel{\mathrm{N}}{\mathrm{S}_{2}}+\mathrm{N}$ & $\begin{array}{ll}\text { No } & \left(S_{2}\right) \\
\text { Yes } & \left(S_{2}\right)\end{array}$ \\
\hline Monaural XOR & & $\begin{array}{l}N \\
S_{1}+N \\
S_{2}+N\end{array}$ & $\begin{array}{l}\mathbf{N} \\
\mathbf{N} \\
\mathbf{N}\end{array}$ & $\begin{array}{l}\text { Neither } \\
\mathrm{S}_{1} \\
\mathrm{~S}_{2}\end{array}$ \\
\hline Dichotic XOR & & $\begin{array}{l}\mathrm{N} \\
\mathrm{S}_{1} \\
\mathrm{~N}\end{array}$ & $\begin{array}{l}\mathbf{N} \\
\mathbf{N} \\
\mathbf{S}_{2}+\mathbf{N}\end{array}$ & $\begin{array}{l}\text { Neither } \\
\mathrm{S}_{1} \\
\mathrm{~S}_{2}\end{array}$ \\
\hline
\end{tabular}

Note-All conditions include uncorrelated noise in the contralateral earphone. $S_{1}$ is a $630-\mathrm{Hz}$ sinusoid: $S_{2}$ is a $1400 \mathrm{~Hz}$ sinusoid.

\section{Procedure}

Three female college students with normal hearing served as $O s$ in the experiment. The Os were highly practiced in two-channel detection tasks. They were paid an hourly wage plus a bonus based upon their individual performance. The Os were housed in a single-walled Industrial Acoustics chamber inside a separate acoustically insulated room. The general procedure has been described in a previous report (Sorkin, Pastore, \& Gilliom, 1968). Continuous background noise in each channel was generated by two Elgenco noise generators (Model 602A). The noise and signals were bandlimited only by the amplifier-transformer-headphone [ $\mathrm{Scott}, 299 \mathrm{~T}$, (2) TDH-39(300)] combination. The background noise in each channel was fixed throughout the experiment at an overall level of approximately $62 \mathrm{~dB}$ re .0002 microbar. The signals were generated by Hewlett-Packard Model 200 ABR oscillators; all signals were $100 \mathrm{msec}$ in duration, gated on and off at positive zero-crossings with less than a one-cycle rise/decay.

For each O-ear-frequency combination, two signal levels were employed, a level equal to a d' of about 1.0 and a level equal to a $\mathrm{d}^{\prime}$ of about 2.0. Earphone signal voltages ranged from 0.45 to
$0.9 \mathrm{mV}$; for the level of continuous noise employed $(10 \mathrm{mV}$ overall) signals of 0.45 and $0.9 \mathrm{mV}$ correspond to $E / N o$ values of 3.78 and 15.1 , respectively. We used different signal levels for each frequency and each $O$ in an attempt to match the detectabilities of the signals in different frequencies and channels. In this manner, we hoped to minimize the possibility that the $O$ would attend only to the most easily detectable signal. Because of practical limitations, we had to settle for levels which were only approximately equal in detectability.

The basic experimental procedure was a standard single-interval yes/no task. Trial events consisted of a visually marked warning interval $(200 \mathrm{msec})$, a blank interval ( $600 \mathrm{msec})$, the observation interval $(100 \mathrm{msec})$, a response and feedback interval $(2,600 \mathrm{msec}$ in the single-frequency tasks and $3,100 \mathrm{msec}$ in the XOR tasks), and an intertrial interval $(1,500 \mathrm{msec})$. Trials were run in 100 -trial blocks with a short rest period between blocks. Six to eight blocks were run during each daily 2 -h session.

In the single-frequency conditions, the $O$ had to detect the presence of the signal in one ear; the other ear contained uncorrelated noise. The probability of signal occurrence was 0.5 . In the monaural XOR condition, uncorrelated noise was presented in the right (nonsignal) ear, and a $630-\mathrm{Hz}$ signal, a $1,400-\mathrm{Hz}$ signal, or neither signal could occur in the left channel. The $O$ had to make one of three pushbutton responses to indicate whether "no signal," " $630-\mathrm{Hz}$ signal," or " $1,400-\mathrm{Hz}$ signal" was present. The probability of occurrence of each of these events was $1 / 3$. In the dichotic version of the XOR, the occurrence of the $630-\mathrm{Hz}$ signal was limited to the left earphone channel, and the $1,400-\mathrm{Hz}$ signal was limited to the right channel. The $O$ had to indicate which of the three events had occurred. The probability of occurrence of each of these events was $1 / 3$. Uncorrelated noise was present in both earphones.

\section{RESULTS}

The data from the single-frequency conditions consisted of hit and false alarm probabilities for each $\mathrm{O}$, ear, signal level, and signal frequency. The computed d's (averaged across days) are tabled in the first two columns of Tables 2-5. Except as noted in the tables, each of these entries is based on at least 800 trials. The entries in the first column of each table indicate left-ear

Table 2

Performance (d') for Single-Channel (Control) Conditions for All Os; Parameter (d') Estimates (See Text) and Chi-Square Values for Free SCO Model

\begin{tabular}{|c|c|c|c|c|c|c|c|}
\hline \multirow[b]{2}{*}{$\mathrm{O}$} & \multirow{2}{*}{$\begin{array}{c}\text { XOR } \\
\text { Condition } t\end{array}$} & \multicolumn{2}{|c|}{$\begin{array}{l}\text { Obtained Single- } \\
\text { Channel } \mathrm{d}^{\prime} \mathrm{s}\end{array}$} & \multicolumn{2}{|c|}{$\begin{array}{l}\text { Estimated Single- } \\
\text { Channel d's }\end{array}$} & \multicolumn{2}{|c|}{ Chi Square (df) } \\
\hline & & $630 \mathrm{~Hz}$ & $1400 \mathrm{~Hz}$ & $630 \mathrm{~Hz}$ & $1400 \mathrm{~Hz}$ & $630 \mathrm{~Hz}$ & $1400 \mathrm{~Hz}$ \\
\hline LW & $\begin{array}{l}\text { HM } \\
\text { LM } \\
\text { HD } \\
\text { LD }\end{array}$ & $\begin{array}{r}1.81 \\
.92 \\
1.81 \\
.92\end{array}$ & $\begin{array}{l}2.68 \\
1.42 \\
2.40 \\
1.22\end{array}$ & & $\begin{array}{l}2.14 \\
1.50 \\
2.13 \\
1.37\end{array}$ & & $\begin{array}{r}392.6 * *(9) \\
133.7 * *(9) \\
252.0 * *(6) \\
44.2 * *(6)\end{array}$ \\
\hline
\end{tabular}

$f_{H}, L, M$, and $D$ represent. respectively, high signal level, low signal level, monaural task, and dichotic task. + tiased on only 400 trials $*_{p}<.05 \quad *^{*} p<.01$ 
Table 3

Performance (d') for Single-Channel (Control) Conditions for All Os; Parameter (d') Estimates (See Text) and Chi-Square Values for Free SDO Model; Chi-Square Values for Fixed SDO Model

\begin{tabular}{|c|c|c|c|c|c|c|c|}
\hline \multirow[b]{2}{*}{$\mathrm{O}$} & \multirow{2}{*}{$\begin{array}{c}\text { XOR } \\
\text { Conditiont }\end{array}$} & \multicolumn{2}{|c|}{$\begin{array}{l}\text { Obtained Single- } \\
\text { Channel d's }\end{array}$} & \multicolumn{2}{|c|}{$\begin{array}{c}\text { Estimated Single- } \\
\text { Channel d's }\end{array}$} & \multicolumn{2}{|c|}{ Chi Square (di) } \\
\hline & & $630 \mathrm{~Hz}$ & $1400 \mathrm{~Hz}$ & $630 \mathrm{~Hz}$ & $1400 \mathrm{~Hz}$ & Free & Fixed \\
\hline LW & $\begin{array}{l}\text { HM } \\
\text { LM } \\
\text { HD } \\
\text { LD }\end{array}$ & $\begin{array}{r}1.81 \\
.92 \\
1.81 \\
.92\end{array}$ & $\begin{array}{l}2.68 \\
1.42 \\
2.40 \\
1.22\end{array}$ & $\begin{array}{r}1.34 \\
.86 \\
1.76 \\
.64\end{array}$ & $\begin{array}{l}1.58 \\
1.26 \\
1.58 \\
1.28\end{array}$ & $\begin{array}{r}307.7 * *(6) \\
99.6 * *(6) \\
138.0 * *(4) \\
48.2 * *(4)\end{array}$ & $\begin{array}{r}1378.4 * *(12) \\
280.8 * *(12) \\
437.1 * *(8) \\
143.4 * *(8)\end{array}$ \\
\hline
\end{tabular}

†H, L. M. and $D$ represent, respectively, high signal level, low signal level, monaural task, and dichotic task.

$$
\div \text { Based on only } 400 \text { trials } \quad{ }^{*} p<.05 \quad{ }^{* *} p<.01
$$

$(630 \mathrm{~Hz})$ detection performance, while the entries in the second column indicate left-ear $(1,400 \mathrm{~Hz})$ or right-ear $(1,400 \mathrm{~Hz})$ performance, depending on whether the related XOR condition was monaural (HM and LM in the tables) or dichotic (HD and LD in the tables). ${ }^{2}$

The data from the XOR conditions consisted of a 9-entry confusion matrix for each condition ( $M$ or $D)$, signal level ( $\mathrm{H}$ or $\mathrm{L}), \mathrm{O}$, and session. Two or three sessions were devoted to each condition at each signal level and were run successively. We have fit each model to each of the obtained XOR matrices. A grid-search method was used to determine the parameter values which yielded a minimum chi-square value (Atkinson, Bower. \& Crothers, 1965). The d's and $\beta$ s were allowed to vary between zero and four. ${ }^{3}$ The $\alpha$ in the SCO model was allowed to vary between zero and one. For the TCU, TCF, and SDO models, two types of fits were made for each data matrix. In the first of these, the free fit, the $\mathrm{d}^{\prime} \mathrm{s}$ and $\beta \mathrm{s}$ were allowed to vary: in the second, the fixed fit, the d's were fixed at the obtained single-channel values and only the $\beta$ s were allowed to vary. The resultant $d^{\prime}$ estimates were averaged over the two or three sessions (at each condition) that each $O$ ran. These averages are listed in the third and fourth columns of Tables 2-5. The chi-square values for each session (and model) were summed and are listed in the last two columns of Tables 2.5.4 We have not tabled any of the estimated $\beta$ values; however, these were all reasonably consistent with the single-channel values.

An assumption of the SCO model is that the $\mathrm{O}$ attends to his more sensitive channel. Thus, the model requires a computational definition of "more sensitive." We chose a difference of $0.2 \mathrm{~d}^{\prime}$ units on the single-frequency conditions as our definition. For those

Table 4

Performance (d') for Single-Channel (Control) Conditions for All Os; Parameter (d') Estimates (See Text) and Chi-Square Values for Free TCU Model; ChiSquare Values for Fixed TCU Model

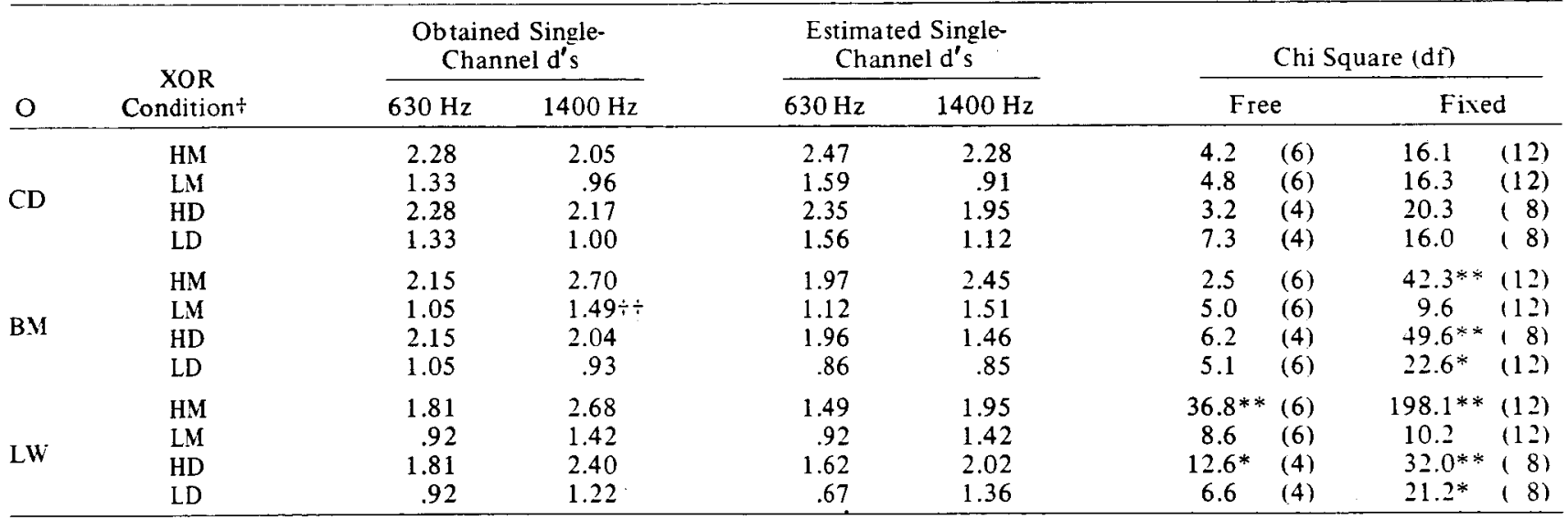

${ }^{+} H, L, M$. and $D$ represent, respectively, high signal level, low signal level, monaural task, and dichotic task. t fBased on only 400 trials $\quad{ }^{*} p<.01 \quad{ }^{* *} p<.01$ 
Table 5

Performance $\left(d^{\prime}\right)$ for Single-Channel (Control) Conditions for All Os; Parameter ( $d^{\prime}$ ) Estimates (See Text) and Chi-Square Values for Free TCF Model: Chisquare Values for Fixed TCF Model

\begin{tabular}{|c|c|c|c|c|c|c|c|}
\hline \multirow[b]{2}{*}{0} & \multirow{2}{*}{$\begin{array}{c}\text { XOR } \\
\text { Condition } \div\end{array}$} & \multicolumn{2}{|c|}{$\begin{array}{c}\text { Obtained Single- } \\
\text { Channel } \mathrm{d}^{\prime} \mathrm{s}\end{array}$} & \multicolumn{2}{|c|}{$\begin{array}{c}\text { Estimated Single- } \\
\text { Channel d's }\end{array}$} & \multicolumn{2}{|c|}{ Chi Square (df) } \\
\hline & & $630 \mathrm{~Hz}$ & $1400 \mathrm{~Hz}$ & $630 \mathrm{~Hz}$ & $1400 \mathrm{~Hz}$ & Free & Fixed \\
\hline $\mathbf{L} \mathbf{W}^{\top}$ & $\begin{array}{l}\text { HM } \\
\text { LM } \\
\text { HD } \\
\text { LD }\end{array}$ & $\begin{array}{r}1.81 \\
.92 \\
1.81 \\
.92 \\
\end{array}$ & $\begin{array}{l}2.68 \\
1.42 \\
2.40 \\
1.22\end{array}$ & $\begin{array}{r}1.74 \\
1.00 \\
1.77 \\
.79 \\
\end{array}$ & $\begin{array}{l}2.30 \\
1.53 \\
2.21 \\
1.45 \\
\end{array}$ & $\begin{array}{c}7.1 \quad(6) \\
6.7 \quad(6) \\
11.0^{*}(4) \\
13.5^{* *}(4)\end{array}$ & $\begin{array}{l}43.5 * *(12) \\
11.3 \quad(12) \\
14.2 \quad(8) \\
26.2 * *(8)\end{array}$ \\
\hline
\end{tabular}

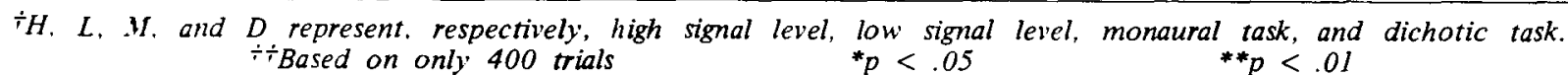

Os and conditions with smaller differences, the fit was made twice-one time using each of the channels as the more sensitive channel. Both of these fits are listed in Table 2.

Examination of the resulting chi-square statistics indicates that the single-channel models provide the poorest fits of any of the models. Table 6 lists the chi-square statistics summed over conditions for each $O$. The fit of the SDO model is better than the SCO, but both can be safely rejected. The TCF and TCU fare much better, and the TCU model is acceptable, for two of the three Os. Examination of Table 4 shows that the TCU model is rejected in only 2 of the 12 free-fit conditions. Furthermore, in the free parameter applications of the TCU and TCF models, the computed $d^{\prime}$ values are in close agreement with the d's of the single-channel control conditions.

\section{DISCUSSION}

We did not include a summary of the $d^{\prime}$ or chi-square values for the SCM model in the Results section. This is because the SCM model is simply a TCU model with scaled down values of $d^{\prime}$ in each channel. Thus, a free parameter fit of the SCM model would yield the same fit as the TCU model. We noted that the TCU's estimates of the $d^{\prime}$ values were quite close to the single-channel values. Since the SCM fixed-d'values are scaled $\sqrt{2}$ from the single-channel values, we would expect that a fixed parameter fit of SCM would be very poor. Thus, performance in the XOR task is much better than predicted by the SCM model, and we may safely reject it in favor of TCU. One may also conclude that for the criterion values employed by these Os, TCF is not a very good approximation to the TCU model. An even stronger test between the TCU and TCF models would be to force the Os to employ very liberal criteria for responding "signal" in each channel. This could have been accomplished by manipulation of the pay-off matrix and/or the signal presentation probabilities.

The TCU model does not incorporate any significant departure from existing models of $O$ behavior in single-channel auditory detection. Thus, in the XOR task involving simple types of auditory signals and highly trained Os, there is no evidence for either cross-channel interference or a limited processing capacity. We have argued that our hypothesized two-channel $O$ should not be vulnerable to cross-channel interruptions in the XOR task; the present result is consistent with that hypothesis. Furthermore, we may argue that performance in some multiple-component signal detection tasks (cf. Green \& Swets, 1966) is also interpretable in these terms: adding signal components to orthogonal signal channels does not improve the detectability of the compound signal according to $\mathrm{d}_{\text {comp }}^{\prime}=\left(\Sigma \mathrm{d}_{\mathrm{i}}^{2}\right)^{1 / 2}$, because cross-channel interference may cause premature termination of signal processing. That is, in multiple frequency experiments, the $O$ attempts to listen simultaneously with several critical bands and can do so effectively on most noise-alone trials. However, when the integration process on any critical band reaches a critical value, processing in the other bands is interrupted and information is lost. This hypothesis is consistent with Ahumada's (1967) filter-bank model.

\section{Table 6}

Chisquare Values for Free Parameter Versions of SCO, SDO, TCU, and TCF Models for All Os, Summed Over All Conditions

\begin{tabular}{|c|c|c|c|c|}
\hline $\mathrm{O}$ & $\mathrm{SCO}$ & SDO & $\mathrm{TCU}$ & TCF \\
\hline CD & $1110.3 * *(30)$ & $381.2 * *(20)$ & (20) & $55.0 * *(20)$ \\
\hline BM & $974.3 * *(33)$ & $373.4 * *(22)$ & $18.8 \quad(22)$ & $71.9 * *(22)$ \\
\hline LW & $822.5 * *(30)$ & $593.5 * *(20)$ & $66.4 * *(20)$ & $38.3 * *(20)$ \\
\hline
\end{tabular}

${ }^{* *} p<.01$ 
We have suggested that at least in two-channel tasks employing relatively simple signals, the two-channel "decrement" is attributable to factors which are present when large observations occur in any channel. There are at least two possible mechanisms for this cross-channel interference. One is strictly on the response side of the system; the other is somewhere on the input side. The first possibility was discussed earlier as being caused by variability in the criterion of a channel. If an $O$ employs a range of different response criteria in a channel that are contingent on the observation in the remote channel, then the variance of his criterion distribution will depend on the variance of the distribution of observations on the remote channel. Furthermore, the different criterion levels will probably not be uniformly distributed over values of the remote observation, and therefore, the magnitude of criterion variability will depend on particular levels of activity in the remote channel. This criterion variability could result in the effective depression of the computed $\mathrm{d}^{\prime}$ for that channel, as a function of the mean level of the observations made on the other channel. The second possible locus of interaction is on the input, e.g., some sort of level-dependent inhibition between the channels. This interpretation is consistent with the models hypothesized to account for decrements in complex two-channel listening tasks. We noted earlier that the particular kinds of cross-frequency and cross-ear interference effects that we reported in our previous studies did not fit within any existing auditory masking framework. In those studies, and in the present experiment, there were no differences between the monaural and dichotic presentation of signals in the two-frequency task (Sorkin, Pohlmann, \& Gilliom, 1973). If there is inhibition between the channels, the site of this inhibition must be at a higher level in the nervous system than that where "conventional" contralateral masking occurs.

Useful applications of the TCU model might be made in XOR tasks involving signals of increased complexity, since this task minimizes the possibility of confounding cross-channel interference and processing-capacity effects. A more general form of the TCU model could be assumed. Let the response strategy of the $O$ be as described by the TCU model but the actual d's be somewhat less than the single-channel d's. An extreme version of this approach is the SCM model in which we assumed the effective $d$ 's to be $1 / \sqrt{2}$ of the single-channel values as a consequence of having only half of the effective signal durations available for processing. In general, one could weight the single-channel $\mathrm{d}^{\prime}$ values of the TCU model by $1 / \mathrm{k}$ where $(1 / \sqrt{2})<k<1$ and apply this model to the XOR task (allowing $\mathrm{k}$ and the $\beta$ s to vary). If we had done so in the present experiment, we would have arrived at an estimated $\mathrm{k}$ of approximately 1 , indicating simultaneous two-channel processing. It is likely that in an XOR version of a word detection task such as that employed by Moray and OBrien (1967), $\mathrm{k}$ would be much smaller, perhaps less than $1 / \sqrt{2}$.

Another potential application of the model is in assessing behavior in sequential types of "two-channel" tasks. Such tasks have not been consistently identified as two-channel tasks since it is not clear that they require the simultaneous processing of inputs. Gilliom (1972), in work done in our laboratory, reported on tasks which require the processing of different frequency signals occurring (independently) in two temporally separated intervals. He reported interference effects quite similar to those observed in simultaneous inclusive-or detection tasks. We propose that interference between the observations of a sequential XOR task might reveal the time course of such interactions relatively free from interactions due to cross-channel interference.

\section{APPENDIX}

The density function for the observations, z, given noise alone in that channel is

$$
f(z \mid N)=\left[1 /(2 \pi)^{1 / 2}\right] \exp \left(-z^{2} / 2\right)
$$

For clarity let

$$
f(z)=f(z \mid N)
$$

and for the SCO, TCU, and TCF Os,

$$
f\left(z \mid S_{i}\right)=f\left(z-d_{i}\right),
$$

for the SDO and SCM Os,

$$
f\left(z \mid S_{i}\right)=f\left(z-d_{i} / \sqrt{2}\right),
$$

where $d_{i}$ is the single-channel detectability $\left(d^{\prime}\right)$ of the signal. Also let

$$
F(\gamma)=\int_{-\infty}^{\gamma} f(z) d z
$$

(1) Single-channel $O\left(d_{1}, \beta_{1}, \alpha\right)$ :

Let $d_{1}$ be the detectability of the more sensitive channel. Then,

$$
\begin{aligned}
& P\left(S_{1} \mid S_{1}\right)=1-F\left(\beta_{1}-d_{1}\right) \\
& P\left(S_{1} \mid S_{2}\right)=1-F\left(\beta_{1}\right) \\
& P\left(S_{1} \mid N\right)=1-F\left(\beta_{1}\right) \\
& P\left(S_{2} \mid S_{2}\right)=\alpha F\left(\beta_{1}\right) \\
& P\left(S_{2} \mid S_{1}\right)=\alpha F\left(\beta_{1}-d_{1}\right) \\
& P\left(S_{2} \mid N\right)=\alpha F\left(\beta_{1}\right) \\
& P(N \mid N)=(1-\alpha) F\left(\beta_{1}\right) \\
& P\left(N \mid S_{1}\right)=(1-\alpha) F\left(\beta_{1}-d_{1}\right)
\end{aligned}
$$




$$
\mathrm{P}\left(\mathcal{N} \mid \mathrm{S}_{2}\right)=(1-\alpha) \mathrm{F}\left(\beta_{1}\right)
$$

(2) Same-Different $O\left(d_{1}, d_{2}, \beta_{1}, \beta_{2}\right)$ :

$$
\begin{aligned}
& \mathrm{P}\left(\mathrm{S}_{1} \mid \mathrm{S}_{1}\right)=\mathrm{F}\left[\left(\mathrm{d}_{1} / \sqrt{2}\right)-\beta_{1}\right] \\
& \mathrm{P}\left(\mathrm{S}_{1} \mid \mathrm{S}_{2}\right)=1-\mathrm{F}\left[\left(\mathrm{d}_{2} / \sqrt{2}\right)+\beta_{1}\right] \\
& \mathrm{P}\left(\mathrm{S}_{1} \mid \mathrm{N}\right)=1-\mathrm{F}\left(\beta_{1}\right) \\
& \mathrm{P}\left(\mathrm{S}_{2} \mid \mathrm{S}_{2}\right)=\mathrm{F}\left[\left(\mathrm{d}_{2} / \sqrt{2}\right)-\beta_{2}\right] \\
& \mathrm{P}\left(\mathrm{S}_{2} \mid \mathrm{S}_{1}\right)=1-\mathrm{F}\left[\left(\mathrm{d}_{1} / \sqrt{2}\right)+\beta_{2}\right] \\
& \mathrm{P}\left(\mathrm{S}_{2} \mid \mathrm{N}\right)=1-\mathrm{F}\left(\beta_{2}\right) \\
& \mathrm{P}(\mathrm{N} \mid \mathrm{N})=\mathrm{F}\left(\beta_{1}\right)+\mathrm{F}\left(\beta_{2}\right)-1 \\
& \mathrm{P}\left(\mathrm{N} \mid \mathrm{S}_{1}\right)=\mathrm{F}\left[\left(\mathrm{d}_{1} / \sqrt{2}\right)+\beta_{2}\right]-\mathrm{F}\left[\left(\mathrm{d}_{1} / \sqrt{2}\right)-\beta_{1}\right]
\end{aligned}
$$

$P\left(N \mid S_{2}\right)=F\left[\left(d_{2} / \sqrt{2}\right)+\beta_{1}\right]-F\left[\left(d_{2} / \sqrt{2}\right)-\beta_{2}\right]$

(3) Two-Channel Unweighted $O\left(d_{1}, d_{2}, \beta_{1}, \beta_{2}\right)$ :

$$
\begin{aligned}
P\left(S_{1} \mid S_{1}\right)= & \text { Prob. }\left[\left(x>\beta_{1}, y<\beta_{2}\right) \mid S_{1}\right] \\
& + \text { Prob. }\left[\left(x>\beta_{1}, y>\beta_{2}, x>y\right) \mid S_{1}\right] \\
= & \int_{-\infty}^{\beta_{2}} \int_{\beta_{1}}^{\infty} f\left(x, y \mid S_{1}\right) d x d y \\
& +\int_{\beta_{2}}^{x+\beta_{2}-\beta_{1}} \int_{\beta_{1}}^{\infty} f\left(x, y \mid S_{1}\right) d x d y \\
= & \int_{-\infty}^{\beta_{2}} f(y) d y \int_{\beta_{1}}^{\infty} f\left(x-d_{1}\right) d x \\
& +\int_{\beta_{1}}^{\infty} f\left(x-d_{1}\right) \int_{\beta_{2}}^{x+\beta_{2}-\beta_{1}} f(y) d y d x \\
= & F\left(\beta_{2}\right)\left[1-F\left(\beta_{1}-d_{1}\right)\right] \\
& +\int_{\beta_{1}}^{\infty} f\left(x-d_{1}\right)\left[F\left(x+\beta_{2}-\beta_{1}\right)-F\left(\beta_{2}\right)\right] d x
\end{aligned}
$$

then,

$$
P\left(S_{1} \mid S_{1}\right)=\int_{\beta_{1}}^{\infty} f\left(x-d_{1}\right) F\left(x+\beta_{2}-\beta_{1}\right) d x
$$

\section{Similarly,}

$$
P\left(S_{1} \mid S_{2}\right)=\int_{\beta_{1}}^{\infty} f(z) F\left(z+\beta_{2}-\beta_{1}-d_{2}\right) d z
$$

$$
P\left(S_{1} \mid N\right)=\int_{\beta_{1}}^{\infty} f(z) F\left(z+\beta_{2}-\beta_{1}\right) d z
$$

$$
P\left(S_{2} \mid S_{2}\right)=\int_{\beta_{2}}^{\infty} f\left(z-d_{2}\right) F\left(z-\beta_{2}+\beta_{1}\right) d z
$$

$$
P\left(S_{2} \mid S_{1}\right)=\int_{\beta_{2}}^{\infty} f(z) F\left(z-\beta_{2}+\beta_{1}-d_{1}\right) d z
$$

$$
\mathrm{P}\left(\mathrm{S}_{2} \mid \mathrm{N}\right)=\int_{\beta_{2}}^{\infty} \mathrm{f}(\mathrm{z}) \mathrm{F}\left(\mathrm{z}-\beta_{2}+\beta_{1}\right) \mathrm{d} \mathrm{z}
$$

$$
\mathrm{P}(\mathrm{N} \mid \mathrm{N})=\mathrm{F}\left(\beta_{1}\right) \mathrm{F}\left(\beta_{2}\right)
$$

$P\left(N \mid S_{1}\right)=F\left(\beta_{1}-d_{1}\right) F\left(\beta_{2}\right)$

$\mathrm{P}\left(\mathrm{N} \mid \mathrm{S}_{2}\right)=\mathrm{F}\left(\beta_{2}-\mathrm{d}_{2}\right) \mathrm{F}\left(\beta_{1}\right)$

(4) Two-Channel Flip $O\left(d_{1}, d_{2}, \beta_{1}, \beta_{2}\right)$

$\operatorname{P}\left(S_{1} \mid S_{1}\right)=\operatorname{Prob} .\left[\left(x>\beta_{1}, y<\beta_{2}\right) \mid S_{1}\right]$

$$
\begin{aligned}
& +\frac{1}{2} \text { Prob. }\left[\left(x>\beta_{1}, y>\beta_{2}\right) \mid S_{1}\right] \\
= & {\left[1-F\left(\beta_{1}-d_{1}\right)\right]\left[F\left(\beta_{2}\right)\right] } \\
& +\frac{1}{2}\left[1-F\left(\beta_{1}-d_{1}\right)\right]\left[1-F\left(\beta_{2}\right)\right]
\end{aligned}
$$

$\mathrm{P}\left(\mathrm{S}_{1} \mid \mathrm{S}_{2}\right)=\left[1-\mathrm{F}\left(\beta_{1}\right)\right]\left[\mathrm{F}\left(\beta_{2}-\mathrm{d}_{2}\right)\right]$

$$
+\frac{1}{2}\left[1-F\left(\beta_{1}\right)\right]\left[1-F\left(\beta_{2}-d_{2}\right)\right]
$$

$P\left(S_{1} \mid r\right)=\left[1-F\left(\beta_{1}\right)\right]\left[F\left(\beta_{2}\right)\right]$

$$
+\frac{1}{2}\left[1-F\left(\beta_{1}\right)\right]\left[1-F\left(\beta_{2}\right)\right]
$$

$P\left(S_{2} \mid S_{2}\right)=\left[1-F\left(\beta_{2}-d_{2}\right)\right]\left[F\left(\beta_{1}\right)\right]$

$$
+\frac{1}{2}\left[1-F\left(\beta_{2}-d_{2}\right)\right]\left[1-F\left(\beta_{1}\right)\right]
$$

$P\left(S_{2} \mid S_{1}\right)=\left[1-F\left(\beta_{2}\right)\right]\left[F\left(\beta_{1}-d_{1}\right)\right]$

$$
+\frac{1}{2}\left[1-F\left(\beta_{2}\right)\right]\left[1-F\left(\beta_{1}-d_{1}\right)\right]
$$

$P\left(S_{2} \mid N\right)=\left[1-F\left(\beta_{2}\right)\right]\left[F\left(\beta_{1}\right)\right]$

$$
+\frac{1}{2}\left[1-F\left(\beta_{2}\right)\right]\left[1-F\left(\beta_{1}\right)\right]
$$

$P(N \mid N)=F\left(\beta_{1}\right) F\left(\beta_{2}\right)$

$P\left(N \mid S_{1}\right)=F\left(\beta_{1}-d_{1}\right) F\left(\beta_{2}\right)$

$P\left(N \mid S_{2}\right)=F\left(\beta_{1}\right) F\left(\beta_{2}-d_{2}\right)$ 
(5) Single-Channel Multiplexer $\left(d_{1}, d_{2}, \beta_{1}, \beta_{2}\right)$ :

This $O$ is simply a less efficient version of TCU, e.g., all values of detectability, $d_{i}$, in the TCU model become $\left(d_{i} / \sqrt{2}\right)$. For example.

$P\left(S_{1} \mid S_{1}\right)=\int_{\beta_{1}}^{\infty} f\left[z-\left(d_{1} / \sqrt{2}\right)\right] F\left(z+\beta_{2}-\beta_{1}\right) d z$

\section{REFERENCES}

A humada, A. Detection of tones masked by noise: A comparison of human observers with digital-computer-simulated energy detectors of varying bandwidths. Technical Report No. 29, U.C.L.A. Dept. of Psychology Human Communication Lab.. March 1967.

Atkinson, R. C.. Bower. G. H. \& Crothers. E. J. An introduction to mathematical learning theory. New York: Wiley. 1965

Broadbent, D. E. Decision and stress. New York: Academic Press, 1971.

Eijkman. E., \& Vendrik, A. J. H. Can a sensory system be specified by its internal noise? Journal of the Acoustical Society of America, 1965, 37. 1102-1109.

Garner. W. R.. \& Morton. J. Perceptual independence: Definitions, models, and experimental paradigms. Psychological Bulletin, 1969, 72. 233-259.

Gilliom, J. Binaural detection in a sequential detection paradigm. Journal of the Acoustical Society of America. 1972. 52, 167-168.

Green. D. M.. \& Swets, J. A. Signal detection theory and psychophysics. New York: Wiley. 1966.

Moray, N. Attention: Selective processes in vision and hearing. London: Hutchinson, 1969.

Moray, N. Time sharing in auditory detection: Effect of stimulus duration. Journal of the Acoustical Society of America. 1970a. 47,660-661 (L)

Moray, $\mathrm{N}$. Introductory experiments in auditory time sharing: Detection of intensity and frequency increments. Journal of the Acoustical Society of America, 1970b, 47, 1071-1073.

Moray, N.. \& O'Brien, T. Signal-detection theory applied to selective listening. Journal of the Acoustical Society of America, 1967, 42, 765-772

Pastore, R. E., \& Sorkin. R. D. Simultaneous two-channel signal detection: I. Simple binaural stimuli. Journal of the Acoustical Society of America. 1972, 51, 544-551.

Sorkin, R. D. Extension of the theory of signal detectability to matching procedures in psychoacoustics. Journal of the Acoustical Society of America, 1962, 34, 1745-1751.

Sorkin, R. D. Uncertain signal detection with simultaneous contralateral cues. Journal of the Acoustical Society of America, 1965, 38, 207.212.

Sorkin, R. D., \& Pastore, R. E. Simultaneous binaural signal detection: Comments on "Time sharing in auditory perception." Journal of the Acoustical Society of America. 1971, 49.1319(L).

Sorkin. R. D.. Pastore. R. E., \& Pohlmann. L. D. Simultaneous two-channel sigral detection. II. Correlated and uncorrelated signals. Journal of the Acoustical Society of America. 1972. 51. 1960-1965.

Sorkin, R. D.. Pohlmann, L. D., \& Gilliom. J. D. Simultaneous two-channel signal detection. III. 630 and $1400 \mathrm{~Hz}$ signals. Journal of the Acoustical Society of America. 1973. in press.

Tanner, W. P., Jr. Theory of recognition. Journal of the Acoustical Society of America, 1956, 28, 882-888

Taylor. M. M.. Clarke, D. P. J., \& Smith. S. M. Monaural detection with contralateral cue (MDCC). III. Sinusoidal signals at a constant performance level. Journal of the Acoustical Society of America. 1971.49.1795-1804.

Treisman. A. M. Strategies and models of selective attention. Psychological Review, 1969, 76, 282-299.

Treisman. M. Auditory unmasking. Journal of the Acoustical Society of America. 1963, 35. 1256-1263.

Treisman. M. Detection of binaural tone stimuli: Time sharing or criterion change? Journal of the Acoustical Societs of tmerica. 1972, 51, 625-630.

Treisman, M.. \& Leshowitz, B. The effects of duration, area, and background intensity on the visual intensity difference threshold given by the forced-choice procedure: Derivations from a statistical decision model for sensory discrimination. Perception \& Psychophysics, 1969, 6, 281-296.

Yost. W. A.. Penner. M. J.. \& Feth, L. L. Signal detection as a function of contralateral sinusoid-to-noise ratio. Journal of the Acoustical Society of America, 1972. 51, 1966-1970.

Zinnes. J. L.. \& Kurtz. R. Matching, discrimination, and payoffs Journal of Mathematical Psychology, 1968, 5. 392-421.

Zwislocki. J. J.. Damianopou'os. E. N., Buining. E.. \& Glantz, J. Central masking: Some steady-state and transient effects. Perception \& Psychophỵsics, 1967, 2. 59-64.

\section{NOTES}

1. A response based on such an early decision would not necessarily be correct. but would rarely disagree with the response of an ideal $\mathrm{O}$.

2. The data from the single-frequency conditions were obtained as part of the control conditions of a previously reported experiment (Sorkin, Pohlmann, \& Gilliom. 1973).

3. For the SDO model. d' was allowed to vary between zero and $4 / \sqrt{2}$.

4. Degrees of freedom $=(6$ minus the number of estimated parameters) $x$ (number of matrices obtained at that condition).

5. An alternative approach would be to use the single-channel $\mathrm{d}^{\prime} \mathrm{s}$ and to assume that the decision dimensions were not orthogonal. but were at some angle, $\Theta$, and then estimate $\Theta$ as a parameter in addition to $\beta_{1}$ and $\beta_{2}$. This procedure would involve a more complicated computation than the one proposed.

(Received for publication January 8. 1973: revision received March 19,1973 .) 